\title{
The effects of LSVT BIG home exercises and T'ai Chi on balance and gait in an individual with parkinson's disease: A case study
}

Maureen Romanow Pascal ${ }^{1 *}$, Dayna Ehlers ${ }^{2}$ and Rebecca Hindman ${ }^{2}$

*Correspondence: mpascal@misericordia.edu

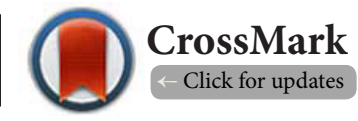

${ }^{1}$ Associate Professor, Physical Therapy Department, Misericordia University, Dallas, PA 18612, USA.

²DPT graduate, Misericordia University, Dallas, PA, 18612, USA.

\begin{abstract}
Background: Parkinson's Disease can lead to deficits that impair quality of life. Common impairments include freezing of gait, balance deficits, and slowed gait speed. There has been limited research done involving the effects of the Lee Silverman Voice Treatment (LSVT) BIG home exercise program (HEP) and T'ai Chi Exercises on an individual with late stage Parkinson's Disease. The purpose of this case study was to determine the effectiveness of T'ai Chi and the LSVT BIG HEP in improving gait and balance deficits in a subject with Parkinson's Disease.

Methods: The subject was an 82-year-old woman with Parkinson's Disease who was classified as Stage 4 on the Hoehn and Yahr scale. She used a power wheelchair independently, and a walker with supervision. The subject participated in an 18-week study with three phases of 6 weeks. Phase A incorporated the LSVT BIG HEP; phase B included a T'ai Chi video program; and Phase C included a combination of both programs. Gait and balance were assessed every six weeks, and after 12 weeks of no intervention.

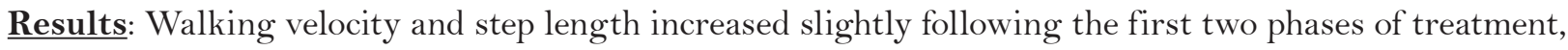
and then declined in the follow-up phase. The subject's Berg Balance score improved from the beginning of the study to the end of Phase B. The subject declined only slightly after twelve weeks without intervention.

Conclusion: This subject with Stage 4 PD demonstrated improvements in balance and gait with supervised exercise that included the LSVT BIG HEP and T'ai Chi exercises. Without intervention, there was a slight decline beyond baseline status. These results indicate a need for more research looking at how exercise affects subjects who are functioning at Hoehn and Yahr Stage 4. Continued supervised exercise may help maintain or improve function in the later stages of Parkinson's disease.
\end{abstract}

Keywords: Parkinson's Disease, LSVT BIG, Tai Chi, Parkinson's, high amplitude exercise, case study

\section{Introduction}

Parkinson's disease (PD) affects as many as 10 million people worldwide, with an economic burden of at least 14.4 billion dollars a year in the United States [1]. Persons with PD need specific visual, verbal, and tactile cueing to initiate the proper neuromuscular response for normal movement. Recent research suggests exercise can encourage neuroplasticity after diagnosis, and higher training intensities may be a more appropriate intervention to see lasting effects [2-7]. Two approaches that have been used to help with PD are LSVT (Lee Silverman Voice Training) BIG and T'ai Chi $[2,4,6]$.

LSVT BIG is a program performed by certified therapists. The intensive four-week program encourages increased amplitude of movement during exercise and functional activities. Since stepping strategies and reaching motions are usually perceived to be bigger than they actually are by people with 
$\mathrm{PD}$, this program is used in order to make the motions more exaggerated to translate into normal movements $[2,6]$. The goal is to improve perception of movement and promote carryover to daily activities. At the conclusion of the program, participants are encouraged to perform LSVT BIG exercises as an ongoing home program. Exercises can be performed using a paper handout or the "Homework Helper" video [8].

T'ai Chi exercises are based on a Chinese philosophy and focus on fluidity of movement. The exercises integrate large and fluid full body motions at a slow speed with emphasis on weight shifting $[4,9]$. This exercise emphasizes on the incorporation of methodical placement of extremities that results in less disjointed movements.

Although both approaches have been shown to improve the symptoms of $P D$, there are currently no research studies that have specifically looked at individuals with the later stages of PD, i.e., stages 4 or 5 on the Hoehn and Yahr Scale [10].

\section{Case presentation}

Participant

At the beginning of our study, the subject had been diagnosed with Parkinson's disease for three years, with initial symptoms of cogwheeling rigidity, small handwriting, and decreased step length with decreased gait speed. She had recently begun living in a long-term care facility due to difficulty with gait and transfers, and was classified as Stage 4 on the Hoehn and Yahr Scale. Our subject ambulated using a U-step walker with supervision, and used a motorized wheelchair independently. She presented with a step-to gait pattern and exhibited difficulty initiating gait, often requiring multiple attempts. She demonstrated slow gait speed with decreased arm swing. She had difficulty with sit to stand transfers secondary to decreased lower extremity strength, balance deficits, and impaired initiation.

She had completed the LSVT BIG program in outpatient physical therapy approximately two weeks prior to the study. The subject gave written consent to participate in a home exercise program using the LSVT BIG Homework Helper and a T'ai Chi video (T'ai Chi for Patients with PD) for our research study. The subject agreed to allow the results of her outpatient therapy assessments to be shared with the researchers as baseline data for our research study. Despite her recent completion of the LSVT BIG program, she required constant cueing to complete the exercises properly due to lack of spatial awareness. She demonstrated difficulty weight-shifting due to her decreased base of support and balance deficiencies. She had decreased upper extremity and lower extremity coordination and required modifications, such as upper extremity support, during exercise to complete all activities.

\section{Intervention}

The intervention consisted of three phases. During each phase, the subject performed exercises five times per week for six weeks, as described below. Balance and gait was assessed after each phase, and again after twelve weeks of no intervention. Gait speed, step length, and stride length were assessed using the Zeno Walkway, a 14-foot electronic walkway with sensors to record the temporal and spatial gait parameters. Sensor information was analyzed using with Protokinetics Motion Analysis Software. The subject used a U-step rolling walker during gait testing. Balance was tested using the Berg Balance Scale (BBS). General function, gait, and balance were also assessed through observations each session.

\section{Phase A}

The subject performed the LSVT BIG HEP using the Homework Helper video, with the researchers providing guarding and verbal cues as needed. This program focused on making "BIG", exaggerated,large amplitude movements. After every session, the subject practiced moving sit to stand 10 times, also using large amplitude movements. She was then encouraged to walk as far as possible before becoming too fatigued or frustrated by freezing of gait.

\section{Phase B}

During Phase B, the subject performed T'ai Chi exercises using a video. The researchers provided guarding and verbal cues as needed. The T'ai Chi exercises focused on weight shifting and full body, slow controlled movements. After the exercises, the subject practiced moving sit to stand and walking, as described in Phase A. Balance and gait assessments were repeated at the end of this phase.

\section{Phase C}

In Phase C, the subject performed a combination of T'ai Chi exercises and LSVT BIG exercises. Each session, the subject had the choice between the two exercise programs. The subject was encouraged to complete both programs fairly equally throughout the week. After the exercises, the subject practiced moving sit to stand and walking, as described in Phase A. Balance and gait assessments were repeated at the end of this phase.

\section{Follow-up}

The follow-up was conducted 12 weeks after no contact with the subject. During that time, she completed a two-week general exercise program with another physical therapist and stated she did occasional seated exercises on her own. Balance and gait assessments were repeated at the end of this phase.

\section{Discussion}

\section{Gait parameters}

Gait velocity and step length improved from the end of Phase A to the end of Phase B, and then declined after 12 weeks of unsupervised exercise (Figure 1). Subjectively, the researchers noted a decline in freezing of gait during intervention, and a return to baseline with no intervention. The mild improvement noted during the intervention may indicate the possibility 
for improved gait with continued intervention. The decline beyond baseline in gait in the absence of regular intervention may show the need for continued activity. In the case of our subject, and all people functioning at Modified Hoehn and Yahr Stage 4 , the ability to continue walking practice is dependent on the availability of supervision. The need for continued assistance with gait may be an important consideration for long-term maintenance of gait mobility and function, and slowing disease progression.

\section{Balance}

The subject demonstrated improvement in two items on the BBS from Phase A to Phase B: standing to sitting and standing unsupported (Figure 2). The overall score on the BBS during the first assessment was $24 / 56$ and improved to $28 / 56$ during the second administration of the test after completing the LSVT BIG HEP. The subject's score did not improve or decline after the other phases of testing.
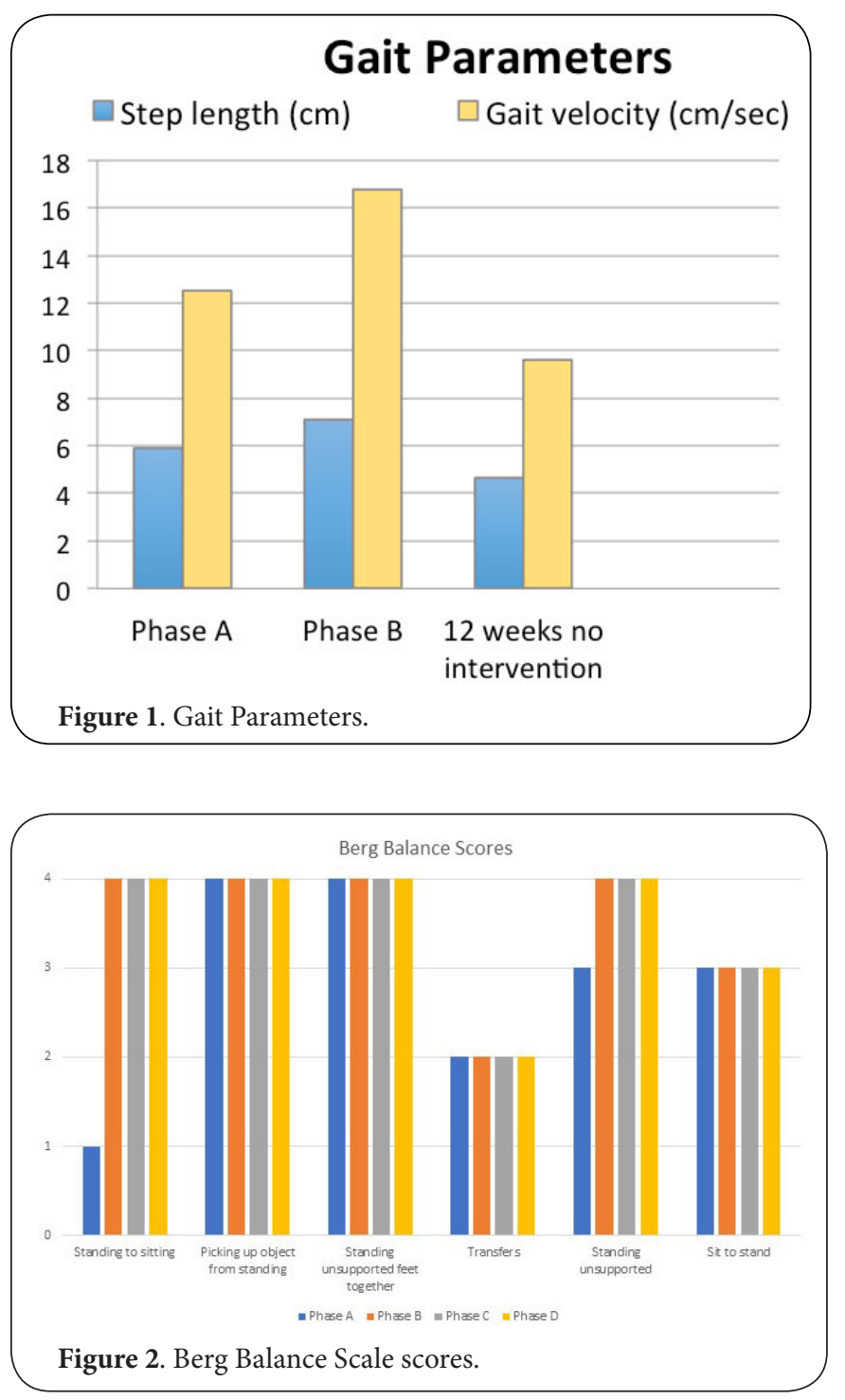

For individuals with PD, the minimal detectable change is a 5 -point increase or decrease. Our subject did not meet this minimal change score, but did maintain the increased score in all subsequent retests of the Berg. The items in which she improved were also items practiced each session, which may indicate the importance of practicing tasks to promote improvement in them. As with gait speed, the improvement in balance is likely to be a factor of continued supervised exercise over 18 weeks. It is encouraging that the subject maintained the gains in balance for at least 12 weeks, even after regular exercise was discontinued.

Although it is clear exercise can be effective and neuroprotective in early and moderate PD, the evidence for more advanced PD remains minimal. A recent systematic review of exercise for PD included no research for people in Stages 4 or 5 [11]. Recently, Studer et al. investigated treadmill training for individuals with advanced PD. Although the study included subjects classified as Stage 4, all of the subjects were able to walk on a treadmill independently, which is unlike the functional level of our subject. There is one published case study that investigated a supervised exercise program of 20 sessions for an individual with advanced PD [12]. There is no published research about the use of regular exercise over a longer period of time.

Unlike the study by Hackney, which required a highly trained instructor and a partner, our study investigated an exercise program that can be implemented with minimal training in any setting. This type of program has the potential to be accessible to more individuals with Stage 4 PD, recognizing the need for supervision as the greatest potential barrier.

\section{Conclusion}

There is currently no research looking at the effects of a supervised home exercise program on improving or maintaining function in people with PD who are Stage 4 on the Hoehn and Yahr scale. This case study demonstrates the possibility of promoting modest improvements and maintaining functional mobility with supervised exercise using the LSVT BIG Homework Helper video, a T'ai Chi exercise video, and supervised practice in transfers and walking. These findings add to the current evidence suggesting exercise helps to slow the neurological decline found in PD. More research is needed to look at the effects of exercise with people in the later stages of PD. The need for supervision should be a consideration when discharging clients from skilled physical therapy services.

\section{List of abbreviations}

BBS: Berg Balance Scale

PD: Parkinson's Disease

LSVT: Lee Silverman Voice Training

HEP: Home exercise program

Competing interests

The authors declare they have no competing interests. 
Pascal et al, Physical Therapy and Rehabilitation 2018,

Authors' contributions
\begin{tabular}{|l|c|c|c|}
\hline Authors' contributions & MP & DE & RH \\
\hline Research concept and design & $\checkmark$ & $\checkmark$ & $\checkmark$ \\
\hline Collection and/or assembly of data & $\checkmark$ & $\checkmark$ & $\checkmark$ \\
\hline Data analysis and interpretation & $\checkmark$ & $\checkmark$ & $\checkmark$ \\
\hline Writing the article & $\checkmark$ & $\checkmark$ & $\checkmark$ \\
\hline Critical revision of the article & $\checkmark$ & $\checkmark$ & $\checkmark$ \\
\hline Final approval of article & $\checkmark$ & $\checkmark$ & $\checkmark$ \\
\hline Statistical analysis & $\checkmark$ & $\checkmark$ & $\checkmark$ \\
\hline
\end{tabular}

\section{Acknowledgement}

The authors would like to thank the Misericordia University Physical Therapy Department for giving us the opportunity to perform this research. This research was partially funded by a Misericordia University Student Research Grant. We dedicate this paper to our subject.

\section{Publication history}

Editor: Catherine Ortega, University of Texas Health Science Center, USA.

Received: 14-Jan-2018 Final Revised: 10-Mar-2018

Accepted: 23-Mar-2018 Published: 03-Apr-2018

\section{References}

1. "Statistics on Parkinson's Disease". Parkinson's Association of the Carolinas. 2017. I Website

2. Farley B, Fox C, Ramig $L$ and McFarland D. Intensive amplitudespecific therapeutic approaches for Parkinson's disease: toward a neuroplasticity-principled rehabilitation model. Top Geriatr Rehabil. 2008; 24:99-114. I Pdf

3. Hirsch MA and Farley BG. Exercise and neuroplasticity in persons living with Parkinson's disease. Eur J Phys Rehabil Med. 2009; 45:215-29. | Article I PubMed

4. Gao Q, Leung A, Yang Y, Wei Q, Guan M, Jia C and He C. Effects of Tai Chi on balance and fall prevention in Parkinson's disease: a randomized controlled trial. Clin Rehabil. 2014; 28:748-753. I Article I PubMed

5. Rahman S, Griffin HJ, Quinn NP and Jahanshahi M. The factors that induce or overcome freezing of gait in Parkinson's disease. Behav Neurol. 2008; 19:127-36. | Article I PubMed Abstract | PubMed FullText

6. Janssens J, Malfroid K, Nyffeler T, Bohlhalter S and Vanbellingen T. Application of LSVT BIG intervention to address gait, balance, bed mobility, and dexterity in people with Parkinson disease: a case series. Phys Ther. 2014; 94:1014-23. | Article I PubMed

7. Schenkman M, Moore CG, Kohrt WM, Hall DA, Delitto A, Comella CL, Josbeno DA, Christiansen CL, Berman BD, Kluger BM, Melanson EL, Jain S, Robichaud JA, Poon C and Corcos DM. Effect of High-Intensity Treadmill Exercise on Motor Symptoms in Patients With De Novo Parkinson Disease: A Phase 2 Randomized Clinical Trial. JAMA Neurol. 2018; 75:219-226. | Article | PubMed

8. Guse L. LSVT BIG Homework Helper. [DVD]. United States: LSVT Global Inc. 2014.

9. Fiore D and Fiore V. Easy Tai Chi-Qigong. [DVD]. United States. 2008.

10. Hoehn MM and Yahr MD. Parkinsonism: onset, progression and mortality. Neurology. 1967; 17:427-42. | PubMed

11. Chung $\mathrm{CL}$, Thilarajah $\mathrm{S}$ and Tan D. Effectiveness of resistance training on muscle strength and physical function in people with Parkinson's disease: a systematic review and meta-analysis. Clin Rehabil. 2016; 30:11-23. | Article | PubMed

12. Hackney ME and Earhart GM. Effects of dance on balance and gait in severe Parkinson disease: a case study. Disabil Rehabil. 2010; 32:679-84. | Article | PubMed Abstract | PubMed FullText
13. Studer V, Maestri R, Clerici I, Spina L, Zivi I, Ferrazzoli D and Frazzitta G. Treadmill Training with Cues and Feedback Improves Gait in People with More Advanced Parkinson's Disease. J Parkinsons Dis. 2017; 7:729739. | Article | PubMed

\section{Citation:}

Pascal MR, Ehlers D and Hindman R. The effects of LSVT BIG home exercises and T'ai Chi on balance and gait in an individual with parkinson's disease: A case study. Phys Ther Rehabil. 2018; 5:1.

http://dx.doi.org/10.7243/2055-2386-5-1 